
allemande

50-1 | 2018

Regards franco-allemands sur la justice dans la construction européenne | Les élections législatives de 2017 en Allemagne et en Autriche

\title{
La CDU/CSU sous Merkel, des partis en phase de modernisation? Les conservateurs allemands, la politique familiale et les enjeux de genre
}

\section{Pierre Baudry}

\section{(2) OpenEdition \\ Journals}

\section{Édition électronique}

URL : https://journals.openedition.org/allemagne/647

DOI : 10.4000/allemagne.647

ISSN : 2605-7913

Éditeur

Société d'études allemandes

\section{Édition imprimée}

Date de publication : 30 juin 2018

Pagination : 131-143

ISSN : 0035-0974

Référence électronique

Pierre Baudry, « La CDU/CSU sous Merkel, des partis en phase de modernisation ? Les conservateurs allemands, la politique familiale et les enjeux de genre ", Revue d'Allemagne et des pays de langue allemande [En ligne], 50-1 | 2018, mis en ligne le 30 juin 2019, consulté le 03 juin 2022. URL : http:// journals.openedition.org/allemagne/647 ; DOI : https://doi.org/10.4000/allemagne.647 


\title{
La CDU/CSU sous Merkel, des partis en phase de modernisation? Les conservateurs allemands, la politique familiale et les enjeux de genre
}

\author{
- Pierre Baudry*
}

Le présent article se propose d'analyser les relations entre les partis démocrateschrétiens et le christianisme dans la décennie 2000-2010 et notamment le lien qu'entretiennent les conservateurs allemands avec une Église catholique fermement attachée à des valeurs conservatrices. Les deux partis démocrates-chrétiens, la Christlichdemokratische Union (CDU) et la Christlich-soziale Union (CSU), puisent en effet leur inspiration dans les principes idéologiques de la doctrine sociale de l'Église catholique malgré leur caractère biconfessionnel. Héritière du catholicisme politique apparu dès le XIX ${ }^{e}$ siècle et de l'expérience œcuménique de rapprochement entre protestants et catholiques pendant l'entre-deux-guerres, la CDU/CSU s'est très tôt imposée sur le Zentrum qui fut refondé dès le lendemain de la Seconde Guerre mondiale. Or, ce qui est saillant pour tout observateur de l'Allemagne des années 2000, ce sont les transformations sociétales importantes qui ont marqué la politique familiale sous Angela Merkel sous l'impulsion de sa ministre de la Famille, Ursula von der Leyen. La question qu'il convient alors de soulever est celle de la mobilisation du référent chrétien et surtout catholique dans un discours et une pratique politique caractérisés par une volonté d'ouverture à de nouveaux modèles familiaux éloignés des schémas conservateurs de l'après-guerre ${ }^{(1)}$.

* Agrégé d'allemand, doctorant au Groupe Sociétés, Religions, Laïcités (GSRL, UMR 8582, EPHE/CNRS).

1 Je tiens à remercier Mme Sylvie Toscer-Angot, maître de conférences HDR à l'Université de Paris-Est Créteil, pour ses précieuses remarques sur la première version de cet article. Je remercie aussi Margot Damiens et Solange Arber, doctorantes à l'Université Paris-Sorbonne, pour leur invitation au séminaire des doctorants du laboratoire junior ELANS/REIGENN (EA 3556, Paris IV) au cours duquel j'ai pu présenter cet article et dont les commentaires ont aussi été très utiles. Le contenu du présent article reste naturellement de ma seule responsabilité. 
Notre hypothèse consiste à avancer que l'élément chrétien fonctionne essentiellement comme un médium ou comme un véhicule d'une culture politique transpartisane, davantage que comme un phénomène confessionnel au sens étroit du terme. C'est plutôt comme le réceptacle de revendications politiques diversifiées (libérale, conservatrice, sociale) que le christianisme opère dans les processus collectifs de culture politique que comme un élément exclusivement religieux. En phase de sécularisation avancée, la mobilisation du christianisme se fait dans l'Allemagne des années 2000 autour d'enjeux de nature autant politique que confessionnelle ${ }^{(2)}$.

\section{La démocratie chrétienne sous Angela Merkel, un parti en phase de recentrement idéologique}

\section{Les réformes libérales dans l'Allemagne des années 2000} et leurs liens avec la politique familiale

Les transformations de la démocratie chrétienne allemande sous l'impulsion d'Angela Merkel s'inscrivent dans une dynamique plus globale d'évolution de l'Allemagne post-réunification. Du point de vue des orientations politiques, on observe en effet une certaine forme de libéralisation dans l'économie comme dans la culture depuis le tournant des années $2000^{(3)}$. Nous privilégierons néanmoins les processus de libéralisation culturelle dans la mesure où l'impact des réformes économiques libérales dans l'Allemagne des années 2000 a souvent été étudié ${ }^{(4)}$.

Par ailleurs, il est courant chez les analystes scientifiques comme chez les commentateurs politiques de prétendre que, depuis son arrivée au pouvoir en 2005, Angela Merkel se serait contentée de poursuivre les réformes entreprises par son prédécesseur. Cette interprétation, exacte dans une grande mesure d'un point de vue économique, ne doit pas faire oublier que les années Merkel sont marquées simultanément par une indéniable modernisation, notamment d'un point de vue sociétal et culturel. Les trois mandats de la chancelière sont en effet caractérisés par des décisions marquantes: projet de sortie définitive de l'énergie nucléaire à partir de 2011, introduction du mariage pour les personnes de même sexe en 2017, accueil d'environ un million de réfugiés syriens et de migrants en 2015-2016.

Notre hypothèse consiste à avancer que les évolutions culturelles de l'Allemagne depuis les années 2000 et la vague de libéralisation de l'économie allemande vont de

2 Sur l'évolution des démocrates-chrétiens, voir Jean-Louis GEORGET, «Les chrétiens-démocrates allemands dans la grande coalition, entre modernitélibérale et pertes de repères ", Revue d'Allemagne et des pays de langue allemande, 40/4, octobre-décembre 2008, p. 553-567; ID., "La démocratie-chrétienne au lendemain de son triomphe: les ambiguïtés d'une incontestable victoire ", in: Les élections fédérales du 22 septembre 2013. Bilans, analyses, perspectives, Hans StARK et Jérôme VAILlant (dir.), Allemagne d'aujourd'hui, n² 206, octobre-décembre 2013, p. 88-101.

3 Sur les mutations des deux Allemagnes depuis la réunification, voir Klaus SCHroeder, Die veränderte Republik. Deutschland nach der Wiedervereinigung, Munich/Berlin, Ernst Vögel Verlag, 2006.

4 La littérature sur les réformes sous Gerhard Schröder est très importante. Voir par exemple le numéro d'Allemagne d'aujourd'hui, Bilan du Gouvernement SPD-Verts du Chancelier Schröder, $\mathrm{n}^{\circ}$ 161, juillet-septembre 2002. On se reportera aussi à René LASSERRE, «Le redressement économique de l'Allemagne», Politique étrangère, 4 (2007) (hiver), p. 803-815, https://www.cairn.info/revue-politiqueetrangere-2007-4-page-803.htm (consulté le 5 janvier 2018). 
pair et qu'elles représentent un ensemble de mutations sociétales plus globales, qui a déjà commencé avec la réunification. L'adaptation aux contraintes de la mondialisation et le renouvellement idéologique des démocrates-chrétiens allemands autour des enjeux sociétaux constituent les deux facettes d'une même libéralisation aux aspects à la fois économiques et culturels. La libéralisation de la société allemande correspond également à des enjeux économiques visant à augmenter le taux d'emploi des femmes face à une pénurie de main-d'œuvre sur le marché du travail. L'idéologie est ici inséparable du calcul économique qui fut aussi déterminant. À cette hypothèse, il convient de plus d'ajouter un intérêt heuristique pour des problématiques sociétales et de libéralisation des mœurs qui ne bénéficient pas du même intérêt que les effets des réformes économiques entreprises depuis Gerhard Schröder. Ces transformations sociétales renvoient entre autres à une nouvelle perception des enjeux idéologiques dans l'Allemagne réunifiée où les anciens conflits idéologiques autour du capitalisme, de la démocratie libérale, de la place de la religion sont remplacés par des attentes plus pragmatiques et centristes.

Nous retiendrons surtout les dossiers qui constituent autant de pierres d'achoppement pour le rapport entre le catholicisme et la CDU. D'abord, c'est l'inflexion des conservateurs allemands sous l'impulsion de la ministre de la Famille, Ursula von der Leyen, qui amena la droite allemande à faire évoluer ses positions ${ }^{(5)}$. Parmi ces réformes, évoquons:

- L'introduction d'un nouveau modèle d'allocations familiales à partir de 2007 sous l'impulsion d'Ursula von der Leyen. Dans le système qui existait depuis 1987 (Erziehungsgeld), les familles pouvaient demander à bénéficier d'une allocation forfaitaire s'étendant sur 24 mois maximum. Ce système avait pour conséquence d'interrompre de manière durable la carrière d'un des parents - généralement celle de la mère - et n'incitait pas les femmes avec de hauts revenus à mettre entre parenthèse leur emploi. Le nouveau système mis en place depuis 2007 dans le cadre de la loi sur l'allocation parentale et sur le congé parental (Gesetz zum Elterngeld und zur Elternzeit) introduit une allocation calculée au prorata du salaire du parent afin d'éviter les pertes financières trop conséquentes et de faciliter la continuité entre activité professionnelle et éducation des enfants. Par ailleurs, le Elterngeld s'étend sur une période maximale de 12 mois voire 14 mois mais uniquement si l'autre parent - en général le père - prend en charge l'éducation de l'enfant sur la période des deux mois supplémentaires.

- Autre initiative phare: le soutien aux crèches publiques dès 2007 avec le projet de créer en tout 230000 places d'ici 2010. Le projet d'Ursula von der Leyen, alors ministre de la Famille, consiste à terme à faire en sorte que 750000 enfants puissent bénéficier d'une place en crèche.

- Enfin, l'ouverture du mariage pour les personnes de même sexe en 2017 marque sans doute une maturation de la société allemande en termes d'égalité des droits et

5 Sur ces questions, voir Nathalie Hillenweck, «La politique familiale d'Ursula von der Leyen: un nouveau modèle de la femme et de la famille entre modernisation et féminisme conservateur? ", in: Serge Gouazé, Anne Salles, Cécile Prat-Erkert (dir.), Les enjeux démographiques en France et en Allemagne: réalités et conséquences, Villeneuve d’Ascq, Presses universitaires du Septentrion, 2011. 
de dépassement de l'idéologie familialiste de l'après-guerre. Le modèle patriarcal et conservateur est dépassé au nom d'une reconnaissance des modèles de vie et familiaux minoritaires.

Les réformes de la politique familiale amenèrent à parler d'un changement de paradigme (Paradigmawechsel) avec le passage d'un État-providence «conservateur» vers un État-providence "social-démocrate» pour reprendre la distinction établie par le sociologue danois Gøsta Esping-Andersen ${ }^{(6)}$. La politique familiale passait en effet d'un modèle fondé sur le maintien de l'enfant - et de la mère - au foyer vers la mise en place de structures étatiques d'accueil, et ce dans un but de faciliter la carrière professionnelle des femmes ${ }^{(7)}$.

Ces réformes, cependant, provoquèrent de vives polémiques aussi bien au sein de la CDU/CSU que de l'Église catholique tandis que l'Église protestante (Evangelische Kirche Deutschland - EKD) exprima une approche de ces questions plus sensible aux innovations introduites par Ursula von der Leyen.

Les critiques vinrent par exemple d'Alois Glück, député et président du parlement bavarois (2003-2008). Il présida par ailleurs de 2009 à 2015 le comité central des catholiques allemands (Zentralkomitee der deutschen Katholiken) qui rassemble les représentants des conseils diocésains, des associations catholiques et les institutions de l'apostolat laïque. Dans une interview pour la Deutschlandfunk, Glück explique ainsi dès février 2007 que: «la politique familiale ne doit pas devenir une dépendance de la politique de l'emploi ${ }^{(8)}$ et considère que la politique familiale d'Ursula von der Leyen privilégie trop les femmes qui travaillent sur les femmes au foyer car le calcul de l'allocation parentale (Elterngeld) dépend du montant du salaire du parent qui en bénéficie ${ }^{(9)}$. Des protestations vinrent d'autres personnalités politiques de la CDU ou de la CSU qui critiquèrent elles aussi ce qu'elles considéraient comme un privilège excessif accordé au modèle familial défendu par von der Leyen. Volker Kauder, député au Bundestag depuis 2005, regretta ainsi dans une interview que le gouvernement ne laisse pas davantage de choix dans les modalités d'éducation insistant par exemple sur le fait qu'il n'y avait rien de conservateur selon lui à ce que les femmes prennent en charge l'éducation des enfants en bas âge ${ }^{(10)}$. Quant à l'évêque catholique d'Augsburg de 2005 à 2010, Monseigneur Walter Mixa, il fut un des critiques les plus fermes de la politique familiale mise en place par von der Leyen. Il avance ainsi que cette politique

6 Gøsta Esping-Andersen, The Three Worlds of Welfare Capitalism, Cambridge, Princeton University Press, 1990.

7 Sur les processus de modernisation des politiques familiales, voir Beate KorTEndieK, «Familie: Mutterschaft und Vaterschaft zwischen Traditionalisierung und Modernisierung», in: Ruth BECKER et Beate Kortendiek (dir.), Handbuch Frauen- und Geschlechterforschung, Wiesbaden, Springer Verlag, $2010^{3}$.

8 "Glück kritisiert Familienpolitik der CDU», interview du 14.02.2007. Texte original: "Die Familienpolitik darf nicht eine Unterabteilung etwa von Arbeitsmarktpolitik sein [...]», http://www. deutschlandfunk.de/glueck-kritisiert-familienpolitik-der-cdu.694.de.html?dram:article_id=64219 (consulté le 20 février 2018).

9 Ibid.

10 Interview de Volker Kauder au Neue Osnabrücker Zeitung, 14.02.2007: «Kauder: Wer beim Baby bleibt, ist nicht altmodisch", https://www.cducsu.de/presse/texte-und-interviews/kauder-werbeim-baby-bleibt-ist-nicht-altmodisch (consulté le 21 février 2018). 
est «nuisible pour les enfants et les familles et vise de manière unilatérale à soutenir de manière active les mères avec des enfants en bas âge » ${ }^{(11)}$.

Les prises de position de l'EKD se distinguent nettement des opinions évoquées plus haut. On se reportera surtout au document intitulé: «Entre autonomie et dépendance - renforcer la famille comme communauté fiable» («Zwischen Autonomie und Angewiesenheit - Familie als verlässliche Gemeinschaft stärken») rédigé sous la présidence de l'ancienne ministre fédérale pour la Famille Christine Bergmann en 2013. Le souci de tenir compte des réalités sociétales se manifeste notamment dans les développements consacrés par l'EKD aux nouveaux défis des familles pour concilier vie professionnelle et vie familiale. L'extrait qui suit montre bien la manière dont les représentants de l'Église protestante perçoivent les enjeux familiaux: "L'introduction d'une allocation parentale calculée selon les revenus est un premier pas vers une nouvelle organisation du partage des tâches entre les conjoints, qui donne aussi aux pères la possibilité s'investir dans l'éducation des enfants lors des premiers mois et qui encourage les femmes actives à avoir des enfants tout en travaillant ${ }^{(12)}$.

La manière dont l'EKD perçoit les crèches publiques apparaît aussi clairement dans le lien qu'il établit entre intégration précoce dans les structures éducatives publiques et le développement cognitif et social des enfants. Insistant sur le retard de l'Allemagne dans la mise en place de politiques publiques actives, le document de l'EKD voit là une cause potentielle d'inégalité sociale ${ }^{(13)}$.

\section{Un recentrement idéologique sur fond de stratégie politique}

À ces évolutions de fond de la politique familiale en Allemagne, il faut ajouter les phénomènes de stratégie politique et idéologique de la part de la chancelière allemande. Le programme économique défendu par Angela Merkel lors de sa première candidature en 2005 avait été clairement libéral et visait une réforme drastique du système fiscal allemand. Mais les contours programmatiques de son projet avaient été ensuite redessinés en faveur d'une approche plus pragmatique et plus moderne d'un point de vue sociétal, qui correspondait à une tendance à la désidéologisation de l'opinion publique allemande ${ }^{(14)}$. Désireuse de mener une stratégie consistant à reprendre des éléments idéologiques venant du SPD, la chancelière a mené une politique rompant

11 Süddeutsche Zeitung du 19.05.2010: «Von der Leyen degradiert Frauen zu Gebärmaschinen». Texte original: «schädlich für Kinder und Familien und einseitig auf eine aktive Förderung der Erwerbstätigkeit von Müttern mit Kleinkindern fixiert», http://www.sueddeutsche.de/politik/bischof-mixakritisiert-familienpolitik-von-der-leyen-degradiert-frauen-zu-gebaermaschinen-1.894163 (consulté le 20 février 2018).

12 EKD, Zwischen Autonomie und Angewiesenheit - Familie als verlässliche Gemeinschaft stärken, 2013. Texte original: «Ein erster Schritt, die Arbeitsteilung zwischen den Partnern neu zu organisieren, ist das vom Einkommen abhängige Elterngeld, das auch Vätern die Möglichkeit eröffnet, sich in den ersten Monaten in die Kindererziehung einzubringen, und berufstätige Frauen ermutigt, Kinder mit ihrer Erwerbstätigkeit zu verbinden.»

13 Ibid.

14 Sur le recentrage des conservateurs allemands, voir Clay Clemens, «Modernisation or Disorientation? Policy Change in Merkel's CDU», German Politics, 2 (2009), n 18. Voir aussi Mariam LAU, Die letzte Volkspartei: Angela Merkel und die Modernisierung der CDU, Stuttgart, Deutsche VerlagsAnstalt, 2009. 
avec certains tabous de son propre camp. En plus des décisions évoquées plus haut, elle soutint ainsi la mise en place d'un salaire minimum (Mindestlohn) comme le demandait depuis longtemps la gauche allemande. Cherchant à se placer clairement au centre idéologique, Merkel tenta de saper le SPD - et y arriva - risquant de laisser émerger une nouvelle force de contestation à sa droite, l'Alternative für Deutschland. Cette politique put fonctionner en raison d'un recentrement général de la société allemande dans les années 2000. En effet, comme l'indiquent Matthias Jung et ses collègues:

«Le système politique de l'Allemagne fédérale et les clientèles politiques de deux grands partis pouvaient susciter jusque dans les années 1980 une loyauté électorale encore relativement stable parmi ses électeurs. Les attaches entre les catholiques pratiquants et les démocrates-chrétiens et les travailleurs appartenant aux syndicats et le SPD trouvaient ses racines dans les Länder de l'Ouest et maintint sa prééminence durant des décennies » ${ }^{(15)}$.

Or, selon une étude d'opinion effectuée sur la population allemande, ce sont ainsi près de $60 \%$ des électeurs qui favorisent des partis centristes a contrario de la polarisation progressisme/conservatisme qui date du XIX ${ }^{\mathrm{e}}$ siècle ${ }^{(16)}$. Toujours selon Matthias Jung et alii, cherchant à la fois des valeurs libérales de développement économique, les électeurs allemands veulent aussi la protection de l'État contre les accidents de la vie: les premières attentes sont traitées par le camp conservateur, les secondes par le $\mathrm{SPD}$, mais sans que les polarisations idéologiques ne puissent totalement répondre à cette tendance ${ }^{(17)}$. La formation de deux grandes coalitions en un peu plus de 10 ans entre 2005 et 2017 alors que l'ancienne RFA n'en avait compté qu'une seule, entre 1966 et 1969 , n'est pas que le signe d'une nécessité politique visant à former une majorité stable, mais aussi le signe d'une évolution des attentes d'un électorat éloigné des grands enjeux idéologiques d'autrefois.

\section{La permanence de la référence chrétienne chez les conservateurs allemands}

\section{Considérations de sociologie électorale: l'attachement aux idées chrétiennes} chez les électeurs conservateurs allemands

Ces remarques, cependant, ne doivent pas cacher la permanence du christianisme et de la doctrine sociale de l'Église catholique sur l'idéologie de la CDU/CSU allemande. Cela reste visible dans les comportements électoraux qui restent nettement en faveur de la CDU/CSU chez les catholiques pratiquants. En 2009, lors des élections fédérales, ce sont $53 \%$ des catholiques des anciens Länder de l'Ouest qui apportèrent leur suffrage aux démocrates-chrétiens, ce chiffre s'élève à $72 \%$ chez les catholiques pratiquants. Chez les protestants, $40 \%$ d'entre eux se décident pour les conservateurs

15 Matthias Jung, Yvonne Schroth, Andrea Wolf, «Angela Merkels Sieg in der Mitte», Aus Politik und Zeitgeschichte, 48-49 (2013), Bundestagswahl 2013. Texte original: "Das Parteiensystem der Bundesrepublik und die interessenpolitischen Ausrichtungen der beiden großen Parteien konnten bis in die 1980er Jahre hinein noch relativ stabile politische Loyalitäten zu Wählergruppen auslösen. Die Bindungen zwischen kirchlich gebundenen Katholiken und der Union sowie gewerkschaftlich organisierten Arbeitern und der SPD waren in den westlichen Bundesländern historisch geprägt und jahrzehntelang von großer Bedeutung.» 
contre $33 \%$ pour les sociaux-démocrates ${ }^{(18)}$. En revanche, toujours en 2009, le SPD n'obtient que $12 \%$ des suffrages chez les catholiques pratiquants.

\section{La permanence du référent confessionnel dans les discours de la CDU/CSU}

Mais à ces analyses démoscopiques il faut adjoindre une étude sur l'idéologie de la démocratie chrétienne et sur les rapports que ce parti biconfessionnel entretient avec la doctrine sociale de l'Église catholique. Cela se laisse attester par l'examen d'un document particulièrement révélateur qui permet d'analyser les points de convergence entre le catholicisme et les démocrates-chrétiens allemands. Il s'agit du Grundsatzprogamm de la CDU de 2007 qui résulta de longs débats internes au sein des conservateurs $^{(19)}$.

Les références au christianisme s'avèrent nombreuses dans ce document et représentent bien plus que des allusions ou un simple rappel d'un «héritage». Il est bien au contraire structurant et traverse de part en part la vision démocrate-chrétienne de la politique formellement caractérisée par trois traits qui se trouvent en lien avec son contenu confessionnel:

- elle est fondée sur une anthropologie;

- elle prend l'éthique comme point d'appui;

- elle constitue une idéologie de synthèse entre conservatisme, libéralisme et pensée sociale dans une forme de centrisme idéologique qu'on retrouve aussi au sein de la doctrine sociale catholique.

Évoquons quelques passages du Grundsatzprogramm autour de trois axes: la revendication d'une identité, d'une anthropologie et même d'une vision du cosmos tirée d'une vision du monde religieuse.

«\$2 Préambule: Une politique démocrate-chrétienne pour l’Allemagne au XXI ${ }^{\mathrm{e}}$ siècle. Nous, démocrates-chrétiens, nous proclamons [wir bekennen uns] nos valeurs et nos objectifs et nous donnons des réponses aux défis de notre temps dans la conscience de notre responsabilité devant Dieu et les hommes par ce programme politique fondamental. [...] Nous nous orientons à l'image chrétienne de l'être humain et de sa dignité intangible [unanstabare Würde] et de là aux valeurs fondamentales de la liberté, de la solidarité et de la justice. $[\ldots] »^{(20)}$.

Quelques pages puis loin, c'est une véritable esquisse de l'anthropologie chrétienne qui est développée et dont les liens avec les revendications relatives aux droits de l'homme méritent d'être relevés:

«L'image chrétienne de l'être humain. $\$ 5$ : Pour nous l'homme a été créé par Dieu à son image [nach seinem Bilde geschaffen]. De cette conception chrétienne de l'Homme il

18 Ibid.

19 CDU/CSU, Grundsatzprogramm, 2007, https://www.cdu.de/system/tdf/media/dokumente/071203beschluss-grundsatzprogramm-6-navigierbar_1.pdf?file=1 (consulté le 21 février 2018).

20 Ibid., «\$2 Präambel: «Christlich demokratische Politik für Deutschland im 21. Jahrhundert. Wir Christliche Demokraten bekennen uns im Bewusstsein unserer Verantwortung vor Gott und den Menschen mit diesem Grundsatzprogramm zu unseren Werten und Zielen und geben Antworten auf die Herausforderungen unserer Zeit. [...] Wir orientieren uns am christlichen Bild vom Menschen und seiner unantastbaren Würde und davon ausgehend an den Grundwerten Freiheit, Solidarität und Gerechtigkeit. [...]» 
s'ensuit que nous proclamons sa dignité inaliénable. La dignité de tout être humain est égale indépendamment de son sexe, de la couleur de sa peau, de sa nationalité, de son âge, de ses convictions religieuses et politiques, du handicap, de sa santé et de ses capacités, de sa réussite ou de ses échecs et du jugement des autres [...]. Dans la conception chrétienne il est responsable devant Dieu et sa conscience, et il est prédisposé à la vie en communauté avec les autres êtres humains» ${ }^{(21)}$.

Cette anthropologie renvoie par ailleurs à une vision de la nature du cosmos imprégnée d'images chrétiennes:

«Menaces sur la création. $\$ 39$ : Dans la vision chrétienne, la création a été confiée à l'homme pour qu'il lui donne forme et veille sur elle. Aujourd'hui un constat s'impose: la création est menacée. $\$ 40$ : De nouvelles évolutions technologiques amènent l'homme à des limites éthiques. Ainsi la recherche biomédicale et en génie génétique contribue de manière essentielle aux soins contre la maladie et contre la douleur. Mais ils contiennent dans le même temps des risques: quand un désir effréné de connaissance et une recherche sans scrupule du profit se combinent, la création et la dignité de l'homme se trouvent en danger. Même le principe de la liberté de la recherche scientifique doit respecter le caractère intangible de la dignité de l'homme et de la création » ${ }^{(22)}$.

Mais cet attachement aux valeurs chrétiennes et conservatrices se trouve encore plus nettement marqué dans le programme fondamental de la CSU (Grundsatzprogramm) de 2016.

«Une politique familiale moderne doit prendre en compte toutes les situations familiales - qu'il s'agisse de la famille classique reposant sur la vie commune avec la mère, le père et les enfants ou les familles monoparentales ou recomposées. Les droits et les devoirs des parents sont prioritaires sur l'action de l'État. Nous ne mettons pas les parents sous tutelle. L'État doit respecter l'autorité éducative [Erziehungshoheit] des parents. Nous refusons une politique pour la société et pour l'éducation qui s’appuie sur l'idéologie du genre et d'une sexualisation précoce» ${ }^{(23)}$.

21 Ibid., «Das christliche Menschenbild $\$ 5$ : Für uns ist der Mensch von Gott nach seinem Bilde geschaffen. Aus dem christlichen Bild vom Menschen folgt, dass wir uns zu seiner unantastbaren Würde bekennen. Die Würde aller Menschen ist gleich, unabhängig von Geschlecht, Hautfarbe, Nationalität, Alter, von religiöser und politischer Überzeugung, von Behinderung, Gesundheit und Leistungskraft, von Erfolg oder Misserfolg und vom Urteil anderer. [...] Er steht nach christlichem Verständnis in der Verantwortung vor Gott und vor seinem Gewissen und ist auf Gemeinschaft mit seinen Mitmenschen angelegt.»

22 Ibid., «Die bedrohte Schöpfung. $\$ 39$ : Die Schöpfung wurde uns nach christlichem Verständnis zur Gestaltung und Bewahrung anvertraut. Heute müssen wir feststellen: Die Schöpfung ist bedroht. 40. Neue technologische Entwicklungen führen den Menschen an ethische Grenzen. So tragen biomedizinische und gentechnische Forschung wesentlich zur Heilung von Krankheiten und Linderung von Leid bei. Sie bergen aber zugleich Risiken: Wenn sich grenzenloser Erkenntnisdrang und gewissenlose Vermarktung verbinden, sind Schöpfung und Menschenwürde in Gefahr. Auch die Freiheit der Forschung hat die Unantastbarkeit der Würde des Menschen und die Schöpfung zu achten.»

23 CSU, Grundsatzprogramm, 2016. Texte original: «Moderne Familienpolitik muss allen familiären Situationen gerecht werden - der klassischen Familie mit der Gemeinschaft von Mutter, Vater und Kindern ebenso wie Eineltern- oder Patchwork-Familien. Rechte und Pflichten der Eltern haben Vorrang vor staatlichem Handeln. Wir bevormunden Eltern nicht. Der Staat muss die Erziehungshoheit der Eltern respektieren. Eine Gesellschafts- und Bildungspolitik, die Gender - Ideologie und Frühsexualisierung folgt, lehnen wir ab», p. 10, http://csu-grundsatzprogramm.de/wp-content/uploads/ CSU_Grundsatzprogramm.pdf (consulté le 21 février 2017). 
Trois valeurs sont exprimées dans ce passage où le normatif et le réalisme se côtoient face aux nouvelles situations sociétales: d'un côté, le principe d'une non-immixtion de l'État dans la vie familiale se voit clairement exprimé au nom du conservatisme qui valorise l'autonomie de la famille par rapport à toute intervention extérieure. D'autre part, les différents types de famille sont reconnus dans leur spécificité sans aucune forme de hiérarchisation entre elles: l'acceptation de la diversité sociétale se trouve ici reconnue. Enfin, on relèvera un ton polémique contre la «théorie du genre», concept répandu dans les milieux conservateurs et visant à rejeter des recherches en sciences sociales sur les problématiques du genre et leurs éventuelles conséquences pratiques en terme de politique publique.

\section{Le marqueur chrétien, un élément de culture politique plus qu'un facteur confessionnel?}

\section{Retour sur l'ethos politique de l'Allemagne d'après-guerre}

Si la permanence d'une idéologie politique qui s'inspire du christianisme doit être expliquée, alors il faut mobiliser d'autres catégories que celles de la sociologie électorale, de l'idéologie politique ou des concepts macro-sociaux (le vieillissement de la population et ses effets sur les comportements électoraux). La permanence doit être expliquée en terme de culture politique, concept à l'intersection dans le champ des études politiques entre étude des partis politiques et étude des institutions politiques.

Par culture politique, nous entendons l'ensemble des représentations collectives qui structurent l'émergence d'un code politique transpartisan comme condition de possibilité de la vie civique d'une communauté politique. On se trouve donc au croisement entre:

- Idéologie: idées et représentations politiques collectives (narratives).

- Effets institutionnels: la culture politique structure un code politique transpartisan avec un impact sur les institutions politiques et l'esprit qui les anime.

- Sociologie politique fondamentale: la culture politique comme condition de possibilité de la vie civique d'une communauté politique.

Le concept de culture politique et d'ethos civique empêche une approche qui corrèle de manière trop restrictive pratique religieuse et choix électoral. On peut même avancer sereinement que la sécularisation de la société n'implique pas le recul du référent confessionnel dans les discours et la pratique politique, mais bien plutôt de nouvelles modalités d'affirmation de la référence religieuse.

Le concept de dignité (Würde) mérite ainsi d'être examiné de manière particulièrement attentive. Il se trouve en effet au croisement entre théologie catholique - thomiste par exemple -, mémoire du totalitarisme et héritage de l'Aufklärung. C'est donc une notion carrefour qui mobilise autant des idéaux politiques modernes de reconnaissance des droits de l'homme que la volonté de proposer une réponse éthique aux affres de l'histoire allemande $\mathrm{du} \mathrm{XX}^{\mathrm{e}}$ siècle. C'est un concept transversal qui renvoie au souvenir du $I I I^{e}$ Reich et de la dictature est-allemande, à la mise en place d'une idéologie subsidiariste, mais aussi aux revendications libérales et d'égalité du XVIII ${ }^{\mathrm{e}}$ siècle.

Mais les origines de ce concept s'avèrent complexes: elles sont à chercher autant dans la théologie chrétienne et la doctrine sociale de l'Église catholique que dans la 
pensée kantienne par exemple. Développé dans la Somme théologique de saint Thomas d'Aquin, le concept de dignité tire ses origines de la culture latine classique chez un Cicéron par exemple. Créé par Dieu selon le théologien catholique, l'homme posséderait une valeur intrinsèque comme résultat de la volonté divine: son importance tiendrait à l'éminente dignité de son créateur qui l'aurait créé à son image.

Mais l'Aufklärung reprit ce concept et y vit un levier de l'autonomisation de la politique par-delà tout référent transcendant: l'homme a en soi une valeur, il n'est pas qu'un être fini et pécheur dépendant de Dieu, mais possède aussi une valeur en soi dans ce monde sans aucun recours à un fondement ontologique ou théologique. Kant utilise même l'idée d'une dignité absolue de l'homme dans une des formulations de l'impératif catégorique même si le lexique de la Würde n'apparaît pas en soi: "Agis de façon telle que tu traites l'humanité, aussi bien dans ta personne que dans toute autre, toujours en même temps comme fin, et jamais simplement comme moyen » ${ }^{(24)}$.

La Würde se présente comme un dispositif sémantique complexe si souple qu'il dépasse le cadre religieux étroit. On le retrouve aussi de manière récurrente - bien qu'avec des inflexions spécifiques - dans le Grundsatzprogramm du SPD qui proclame par exemple: "l'égale dignité de tous les hommes constitue le point de départ et le but de notre politique» ${ }^{(25)}$. Mais, de manière encore plus significative, la notion de Würde se situe au centre du dispositif juridique fondamental de la RFA depuis 1947 et le Préambule qui proclame solennellement:

"Article 1 [Dignité de l'être humain, caractère obligatoire des droits fondamentaux pour la puissance publique]

1) La dignité de l'être humain est intangible. Tous les pouvoirs publics ont l'obligation de la respecter et de la protéger.

2) En conséquence, le peuple allemand reconnaît à l'être humain des droits inviolables et inaliénables comme fondement de toute communauté humaine, de la paix et de la justice dans le monde» ${ }^{(26)}$.

Cet article, qui reprend en partie la déclaration universelle des droits de l'homme de 1948, eut pour fonction de fonder juridiquement l'édifice constitutionnel de l'Allemagne de l'Ouest après les atrocités de la période nationale-socialiste. Il vise à faire de la vie humaine une valeur absolue.

24 Emmanuel Kant, Métaphysique des mours, Paris, Vrin, 1971. Texte original: «Handle so, daß du die Menschheit sowohl in deiner Person, als in der Person eines jeden andern jederzeit zugleich als Zweck, niemals bloß als Mittel brauchest. »

25 Hamburger Programm, Das Grundsatzprogramm der SPD, 2007, p. 13. Texte original: «Die gleiche Würde aller Menschen ist Ausgangspunkt und Ziel unserer Politik», https://www.spd.de/fileadmin/ Dokumente/Beschluesse/Grundsatzprogramme/hamburger_programm.pdf (consulté le 15 février 2018).

26 Grundgesetz, traduction disponible sur le site du Bundestag, https://www.bundestag.de/blob/189762/ f0568757877611b2e434039d29a1a822/loi_fondamentale-data.pdf. Texte original: «I. Die Grundrechte. Artikel 1. 1) Die Würde des Menschen ist unantastbar. Sie zu achten und zu schützen ist Verpflichtung aller staatlichen Gewalt. 2) Das Deutsche Volk bekennt sich darum zu unverletzlichen und unveräußerlichen Menschenrechten als Grundlage jeder menschlichen Gemeinschaft, des Friedens und der Gerechtigkeit in der Welt. » 


\section{Le christianisme en politique, une source de repli identitaire?}

Mais, dans le même temps, les dangers de cette culture christiano-politique s'avèrent évidents: on doit en effet évoquer la récupération identitaire du christianisme autour du concept de Leitkultur ${ }^{(27)}$. Car aux volontés de modernisation de l'appareil idéologique de la CDU/CSU sous Angela Merkel répond aussi une tentation au repli identitaire qui traverse une bonne partie de la droite allemande. On sait que le débat autour de la Leitkultur, ses formes et ses conséquences pour la politique migratoire, fut lancé à l'origine par le président du groupe parlementaire de la CDU/CSU au Bundestag, Friedrich Merz, en l'an 2000 dans une tribune rédigée dans Die Welt. Ce concept réapparaît ensuite dans le Grundsatzprogramm de la CDU qui indique: « $\$ 37$ : Ces valeurs culturelles et ces expériences historiques sont le fondement du maintien dans notre société et forment la culture dominante» ${ }^{(28)}$.

Mais c'est sans doute dans le Grundsatzprogramm de l'Alternative für Deutschland que les développements les plus conséquents sur la Leitkultur sont présents:

«L'Alternative für Deutschland prône une culture de référence allemande, reposant pour l'essentiel sur trois piliers: une tradition religieuse chrétienne, une tradition scientifique et humaniste, dont les racines antiques se sont nourries plus tard des influences de la Renaissance et du Siècle des Lumières, et enfin le droit romain, socle de notre État de droit. [...] L'idéologie du multiculturalisme, au mépris de l'Histoire, met sur le même pied les courants culturels extérieurs et la culture nationale, relativisant ainsi gravement ses valeurs. L'AfD considère cette démarche comme une menace sérieuse pour la paix sociale et pour le maintien de la nation comme entité culturelle. Confrontés à cette menace, l'État et la société civile doivent défendre formellement l'identité culturelle allemande en tant que modèle de référence» ${ }^{(29)}$.

Ce passage est intéressant car il fait des enjeux de culture et d'identité un objet d'action prioritaire pour l'État, qui se voit chargé non pas seulement de protéger la dignité de l'être humain ou des valeurs éthico-politiques, mais une identité nationale substantielle. L'État a une fonction de protection de la culture et non plus seulement de garantie des droits fondamentaux ou de soumission à l'État de droit (Rechtsstaat). L'État se voit pourvu d'un rôle central et chargé d'une mission de défense de la civilisation

27 Sur ces questions, voir notamment Sylvie Toscer-Angot, «Les débats politiques sur la culture de référence [Leitkultur] en RFA au tournant du XXI siècle», Revue d'Allemagne et des pays de langue allemande, 41/2 (2009), p. 199-212.

28 CDU/CSU, Grundsatzprogramm, 2007 (note 19). Texte original: «\$37: Diese kulturellen Werte und historischen Erfahrungen sind die Grundlage für den Zusammenhalt in unserer Gesellschaft und bilden unsere Leitkultur in Deutschland.»

29 Alternative für Deutschland, Grundsatzprogramm, 2016. Texte original: «Die Alternative für Deutschland bekennt sich zur deutschen Leitkultur, die sich im Wesentlichen aus drei Quellen speist: erstens der religiösen Überlieferung des Christentums, zweitens der wissenschaftlich-humanistischen Tradition, deren antike Wurzeln in Renaissance und Aufklärung erneuert wurden, und drittens dem römischen Recht, auf dem unser Rechtsstaat fußt. [...] Die Ideologie des Multikulturalismus, die importierte kulturelle Strömungen auf geschichtsblinde Weise der einheimischen Kultur gleichstellt und deren Werte damit zutiefst relativiert, betrachtet die AfD als ernste Bedrohung für den sozialen Frieden und für den Fortbestand der Nation als kulturelle Einheit. Ihr gegenüber müssen der Staat und die Zivilgesellschaft die deutsche kulturelle Identität als Leitkultur selbstbewusst verteidigen. » 
entendue autant comme culture spécifique (Kultur) que comme ensemble des valeurs fondant la vie commune (Zivilisation).

Les ambiguïtés des références au christianisme en politique ne tiennent pas, pourtant, uniquement à des problématiques de récupération politique d'une culture religieuse, mais peut-être aussi aux ambiguïtés des instances religieuses comme l'Église catholique dans les années 2000. Marqué par le pontificat de Benoît XVI, le catholicisme connaît un moment de réaffirmation identitaire au nom du rejet du "relativisme», de la défense des «racines chrétiennes» de l'Europe, de la stigmatisation de la violence de l'islam. En ce sens, le divorce apparent constaté entre le catholicisme et la CDU allemande autour des enjeux de genre ne doit pas camoufler une nouvelle forme de proximité, mais cette fois entre une droite identitaire et un catholicisme en phase de reconquête.

\section{Conclusion}

En bilan, nous pouvons avancer les éléments suivants. Les mutations sociétales et autour du modèle familial ont été importantes dans l'Allemagne des années 2000 et 2010 et elles ont été menées par ceux qui apparemment étaient les plus éloignés de telles initiatives: les démocrates-chrétiens. Malgré des critiques contre la politique menée par Ursula von der Leyen, celle-ci sut faire évoluer son camp politique sur les problématiques sociétales. Il n'en reste pas moins que l'élément chrétien et les valeurs conservatrices continuent à déterminer la politique et les choix idéologiques de la CDU/CSU autour des enjeux anthropologiques ou culturels.

Notre approche - essentiellement civilisationniste et centrée sur l'Allemagne mériterait sans aucun doute d'être élargie afin d'étudier non seulement les mutations en rapport au catholicisme dans l'Europe au tournant des années 2000-2010, mais aussi les phénomènes de réseaux, de transfert culturel, d'échos des débats politiques et intellectuels au niveau européen. Cela est évident en ce qui concerne les débats autour des «racines chrétiennes» de l'Europe. C'est en effet toute l'Europe qui est concernée et même l'ensemble du monde occidental, dont l'attitude par rapport à la question religieuse bascula à la suite des attentats du 11 septembre et des phénomènes de repli identitaire qui en résultèrent. En ce sens, peut-être que l'Allemagne est un laboratoire politique dont l'intérêt se trouve sous-estimé: les enjeux de démocratisation, de mémoire des totalitarismes et de sécularisation s'y posent de manière au moins aussi aiguë que dans d'autres pays occidentaux. 


\section{Résumé}

Le présent article s'intéresse aux évolutions de la politique familiale sous Angela Merkel. Comment expliquer qu'un parti qui se dit chrétien-démocrate mène une politique familiale qu'on a pu qualifier de "sociale-démocrate»? Comment le parti de la chancelière concilie-t-il adaptation aux évolutions sociétales et conservatisme politique? Partant du concept de culture politique, nous entendons montrer que le caractère chrétien de la CDU/CSU se maintient car il se combine avec la culture politique (ouest)-allemande née après guerre. C'est par l'association entre attachement à la Loi fondamentale (Grundgesetz) et défense de la "dignité» (Würde) de l'être humain que la démocratie chrétienne se modernise afin d'adapter son appareil idéologique aux mutations sociétales.

\section{Zusammenfassung}

Dieser Beitrag befasst sich mit den Entwicklungen der Familienpolitik unter Angela Merkel. Wie kann man erklären, dass eine Partei, die sich christdemokratisch nennt, eine Familienpolitik betreibt, die man als „sozialdemokratisch“ bezeichnen könnte? Wie vereinbart die Partei der Kanzlerin die Anpassung an gesellschaftliche Veränderungen und politischen Konservatismus? Ausgehend vom Begriff der politischen Kultur wollen wir zeigen, dass der christliche Charakter der CDU / CSU erhalten bleibt, weil er sich mit der politischen Kultur (West)Deutschlands, die nach dem Krieg enstanden ist, verbindet. Durch die gleichzeitige Verteidigung der Prinzipien des Grundgesetzes und der "Würde» des Menschen erlebt die christliche Demokratie eine Modernisierungsphase, um ihre Ideologie an die gesellschaftlichen Veränderungen anzupassen.

\footnotetext{
Abstract

This article aims at understanding the evolution of family policy under Angela Merkel. How to explain that a party that calls itself a Christian-democrat leads a family policy that could be described as "social-democratic"? How does the Chancellor's party reconcile adaptation to societal changes and political conservatism? Starting from the concept of political culture, we intend to show that the Christian character of the CDU/CSU is maintained because it merges with the political culture of (West)-Germany born after World War II. It is through the association between the attachment to the fundamental law (Grundgesetz) and the defense of the "dignity" (Würde) of the human being that Christian democracy modernise its political message, but adapts its ideology to societal mutations.
} 